\title{
Neuron-specific expression of p48 Ebp1 during murine brain development and its contribution to $\mathrm{CNS}$ axon regeneration
}

\author{
Hyo Rim Ko ${ }^{1}$, Inwoo Hwang ${ }^{1}$, So Yoon Ahn ${ }^{2,3}$, Yun Sil Chang ${ }^{2,3,4}$, Won Soon Park ${ }^{2,3,4, *}$ E Jee-Yin Ahn ${ }^{1,4, *}$ \\ ${ }^{1}$ Department of Molecular Cell Biology, Center for Molecular Medicine, Samsung Biomedical Research Institute, Sungkyunkwan University \\ School of Medicine, Suwon 16419, ${ }^{2}$ Department of Pediatrics, Samsung Medical Center, Sungkyunkwan University School of Medicine, \\ Seoul 06351, ${ }^{3}$ Stem Cell and Regenerative Medicine Institute, Samsung Medical Center, Seoul 06351, ${ }^{4}$ Department of Health Sciences and \\ Technology, SAIHST, Sungkyunkwan University, Seoul 06351, Korea
}

P48 Ebp1 is expressed in rapidly proliferating cells such as cancer cells and accelerates cell growth and survival. However, its expression pattern and role in central nervous system development have not been studied. Here, we demonstrated the spatiotemporal expression pattern of $\mathbf{p 4 8}$ Ebp1 during embryonic development and the postnatal period. During embryonic development, p48 Ebp1 was highly expressed in the brain. Expression gradually decreased after birth but was still more abundant than $\mathbf{p 4 2}$ expression after birth. Strikingly, we found that p48 Ebp1 was expressed in a cell type specific manner in neurons but not astrocytes. Moreover, p48 Ebp1 physically interacted with beta tubulin but not alpha tubulin. This fits with its accumulation in distal microtubule growth cone regions. Furthermore, in injured hippocampal slices, p48 Ebp1 introduction promoted axon regeneration. Thus, we speculate that p48 Ebp1 might contribute to microtubule dynamics acting as an MAP and promotes CNS axon regeneration. [BMB Reports 2017; 50(3): 126-131]

\section{INTRODUCTION}

The ErbB3 binding protein Ebp1 is a member of the PA2G4 family of proliferation-regulated proteins (1). Despite its initial isolation due to interaction with ErbB3 (2), Ebp1 is expressed in all tissues and cells, including cells that do not express the ErbB3 receptor, such as muscle progenitors (3). Ebp1 is evolutionarily conserved $(1,4)$, suggesting that it functions as a

*Corresponding authors. Jee-Yin Ahn, Tel: +82-31-299-6134; Fax: +82-31-299-6139; E-mail: jeeahn@skku.edu, Won Soon Park, Tel: +82-2-3410-3523; Fax: +82-2-3410-0043; E-mail: wonspark@skku. edu,ws123.park@samsung.com

https://doi.org/10.5483/BMBRep.2017.50.3.190

Received 14 November 2016, Revised 25 November 2016, Accepted 1 December 2016

Keywords: CNS injury, Development, Neuron, P48Ebp1, Regeneration general signaling molecule. The PA2G4 gene encodes two Ebp1 isoforms, p48 and p42. P48 is 54 amino acids longer than $\mathrm{p} 42$ at its $\mathrm{N}$-terminus. The p48 long-form localizes to both the cytoplasm and the nucleolus and suppresses apoptosis, whereas the p42 short-form resides predominantly in the cytoplasm and promotes cell differentiation $(5,6)$. In mammalian cells, p48 is the most common Epb1 form. The p42 isoform is barely detectable because it is polyubiquitinated and subsequently degraded in human cancers (7). In addition, the p42 crystal structure is missing one and a half helices at the amino-terminus, suggesting that this protein is unstable, and that conformational changes associated with 54 fewer amino acid residues account for these possible differential Ebp1 isoform roles (8).

Previously, we showed that the long Ebp1 isoform prevents apoptotic cell death by interacting with nuclear Akt/PKB (protein kinase B) (5) in nerve growth factor (NGF)-treated PC12 cells, fitting with the observation that Ebp1 binds B-cell leukemia/lymphoma2 ( $\mathrm{Bcl} 2)$ mRNA and contributes to $\mathrm{Bcl} 2$ overexpression (9). P48 Ebp1 is highly expressed in proliferating cells such as muscle progenitor cells and in multiple cancer cell lines including glioblastoma, breast cancer, lung cancer, and oral squamous cell carcinoma and promotes cancer cell proliferation and tumor progression (3, 7, 10-12). We have shown that p48 Ebp1 promotes tumor suppressor p53 degradation in human glioma cells and leads to poor clinical outcomes through HDM2 regulation $(10,13)$. Moreover, we showed that cyclin-dependent kinase 2 (CDK2) phosphorylates serine 34 on $\mathrm{p} 48$, which does not exist in p42, and provokes the tumorigenic function of p48 in human cancer cells (11). The shorter p42 isoform has been considered to be a potent tumor suppressor due to its inhibitory growth function $(14,15)$. Very recently, we investigated the molecular mechanisms underlying p42 tumor-suppressing activity in human cancer cells. P42 specifically interacts with the p85 $\mathrm{PI} 3 \mathrm{~K}$ regulatory domain and rapidly reduces $\mathrm{p} 85$ protein levels by linking p85 to HSP70/CHIP-mediated proteaosomal degradation, thereby inhibiting $\mathrm{PI} 3 \mathrm{~K}$ activity in brain tumor cells $(16,17)$.

Although much attention has been devoted to the distinct

ISSN: 1976-670X (electronic edition)

Copyright (C) 2017 by the The Korean Society for Biochemistry and Molecular Biology

(c) This is an open-access article distributed under the terms of the Creative Commons Attribution Non-Commercial License (http://creativecommons.org/licenses/by-nc/4.0) which permits unrestricted non-commercial use, distribution, and reproduction in any medium, provided the original work is properly cited. 
roles of the two Ebp1 isoforms in cellular functions, most of the studies focused on their roles in cancer cells and pathologic conditions. Therefore, little is known about their roles in neural development. Knowledge of the spatial temporal distribution of the two Ebp1 isoforms in the central nervous system (CNS) during development may facilitate the understanding of their function in vivo. Previously we showed p48 Ebp1 is the predominantly expressed isoform in primary cultured hippocampal neurons, promotes neurite outgrowth, and acts as a downstream Trk signaling mediator (18). In this study, we investigated the spatial and temporal expression of p48 and p42 Ebp1 in murine brain. We found that p48 Ebp1 is the predominant isoform expressed during brain development and showed cell type specific expression in neurons but not astrocytes in the cortex and hippocampus. p48 Ebp1 localization to distal microtubules in the growth cone was reflected by its binding to beta tubulin but not alpha tubulin. Importantly, we showed that AAV2-p48 Ebp1 introduction successfully regenerated axon growth after injury in ex vivo hippocampal slice cultures.

\section{RESULTS}

P48 Ebp1 is the major isoform expressed in the developing murine brain

Our previous study indicated that p48 is selectively expressed in the developing brain because rat brain extract from
A

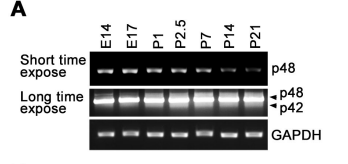

B

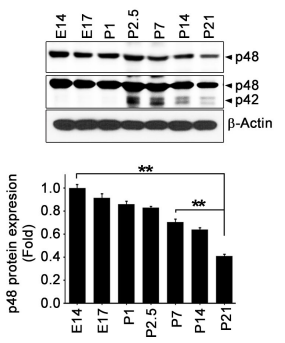

C

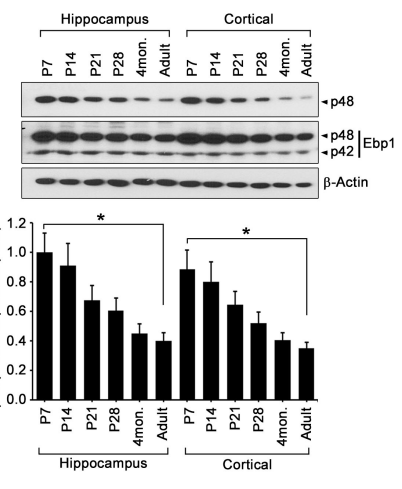

Fig. 1. P48 Ebp1 is a major isoform expressed in the developing murine brain. (A) A series of mouse brain lysates from E14 to P21 were subjected to RT-PCR analysis as indicated. GAPDH was used as an internal control. (B) Immunoblotting analysis was performed against two different antibodies, anti-N-p48 (specific for p48 only) and anti-Ebp1, that recognized a common epitope for p42 and p48 isoforms with a series of mouse brain lysates from E14 to P21 as indicated. (C) Hippocampal and cortical regions were isolated from mouse brain by time and subjected to immunoblotting. $\beta$-actin was used as the loading control. $* P<$ $0.05 ; * * P<0.005$ versus control. Images shown are representative of at least three independent experiments, and each value represents the mean \pm SEM of triplicate measurements. embryonic day (E) 18 and cultured hippocampal neurons showed great $\mathrm{p} 48$ protein abundance, while p42 was almost undetectable (18). To define the expression profiles for the two Ebp1 isoforms during neural development, we conducted RT-PCR and immunoblotting analysis in a series of mouse brains by time: embryonic day $(\mathrm{E}) 14$ through postnatal day $(\mathrm{P})$ 21. p48 Ebp1 mRNA was highly expressed during the embryonic stage and was less abundant after birth but was still expressed at high levels compared to p42 mRNA, which showed little detectable expression in early brain development but was expressed in the postnatal brain (Fig. 1A). We employed two different antibodies, anti-N-p48 (specific for p48 only) and anti-Ebp1, that recognized a common p42 and p48 isoform epitope. Immunoblotting showed that p48 was highly expressed throughout development but was progressively reduced after birth, while p42 began to be visible at P3. This corresponded with our RT-PCR analysis (Fig. 1B). Moreover, in the adult brain, we isolated the hippocampus and cortex and investigated the expression patterns of the two Ebp1 isoforms (Fig. 1C). In both the hippocampus and cortex, although both p48 and p42 proteins were detectable, p48 was the predominant isoform, supporting the hypothesis that p48 is highly involved in cell proliferation.

\section{P48 Ebp1 is mostly expressed in neurons compared to astrocytes in the hippocampus}

To examine cell type p48 expression specificity, we employed an entorhinal cortex through hippocampal $(\mathrm{EH})$ organotypic slice co-culture (OSC), which permitted a well-preserved cytoarchitecture and reflected the corresponding in vivo maturation schedule and connectivity for different anatomical subregions, as well as between-cell functional interactions (19,
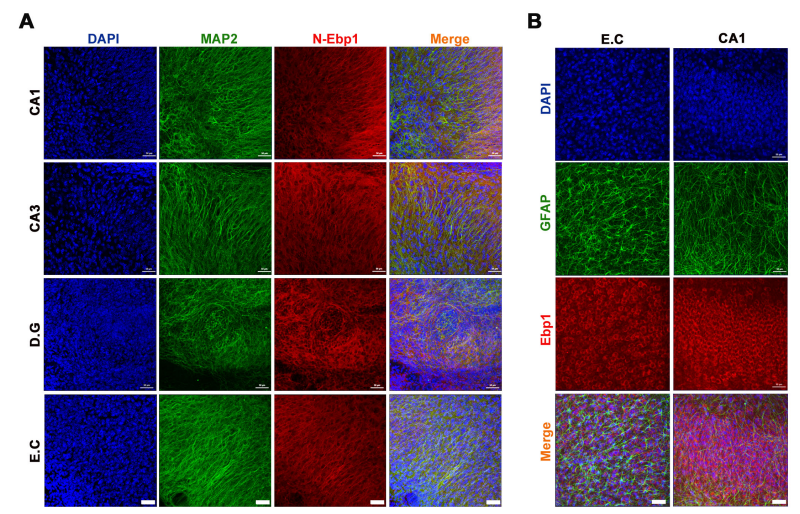

Fig. 2. P48 Ebp1 is mostly expressed in the neurons rather than astrocytes in the hippocampus. The entorhinal cortex through hippocampus $(\mathrm{EH})$ organotypic slices from P7 mice were cultured for 9 days and immunostained. (A) Representative image of neuronal marker MAP2 (green) with p48 Ebp1 in sub-regions of EH-DIV 9 slices. Scale bar, $50 \mu \mathrm{m}$. (B) Representative image of astrocyte marker GFAP (green) with Ebp1 in DIV 9 slices. Scale bar, $50 \mu \mathrm{m}$. 
20). In the experimental condition using P7 mouse brain, we performed $\mathrm{EH}-\mathrm{OSC}$, grew slices in tissue culture for 9 days, and then categorized subregions as entorhinal cortex (EC) and hippocampal CA1, CA3, and dentate gyrus (DG) regions (Supplementary Fig. 1). Immunohistochemical staining demonstrated that p48 Ebp1 (red) was strongly expressed in all regions colocalized with microtubule-associated protein (MAP) 2 (neuron marker; green) (Fig. 2A). Strikingly, the expression patterns of p48 Ebp1 (red) and glial fibrillary acidic protein (GFAP) (astrocyte marker; green) immunofluorescence showed little co-localization in the EC or hippocampus (CA1) (Fig. 2B). Thus, this data suggests that p48 Ebp1 is specifically expressed in neurons rather than astrocytes during brain development.

\section{P48 Ebp1 is present in the growth cone and interacts with microtubules}

In accordance with our findings that p48 Ebp1 was specifically localized throughout all developing hippocampal neurons including the soma and neurites during NGF-mediated PC12 cell differentiation (18), we hypothesized that p48 Ebp1 possesses specific roles in neuronal development. The spatial distribution of p48 Ebp1 was visualized not only in the soma, but also prominently in leading edge neurite precursors during early time points. Both antibodies demonstrated similar Ebp1 distributions, revealing that Ebp1 was found in the soma and proximal axon tips, while its expression tapered off along the
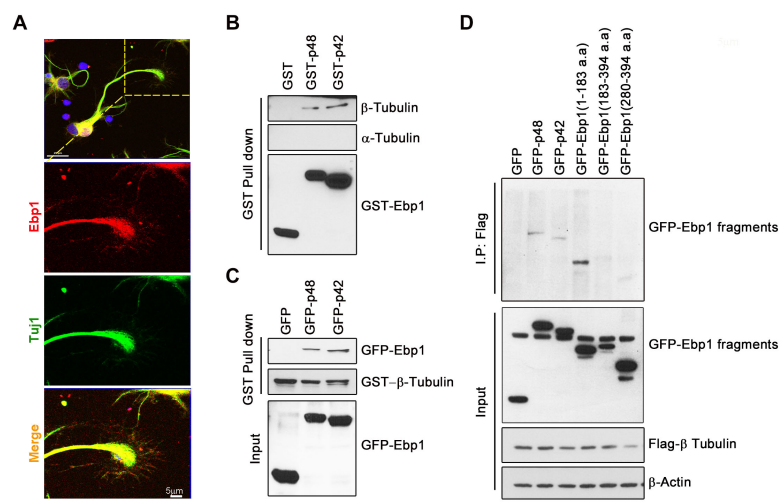

Fig. 3. $\mathrm{P} 48 \mathrm{Ebp} 1$ is present in the growth cone, interacting with microtubules. (A) Cultured neurons were fixed at DIV 3. Neurons were stained with anti-Ebp1 (red) and anti-Tuj1(green). Scale bar, $20 \mu \mathrm{m}$. Representative images at a higher magnification in the region indicated by a box are shown in 2-4 panels. Scale bar, 5 $\mu \mathrm{m}$. (B) GST-pull down assay with purified GST-p48 or p42 and cell lysates from differentiated PC12 cells (DIV) and immunoblotting were performed with indicated antibodies. (C) GST-pull down assay with purified beta tubulin and GFP-p48 orp42 Ebp1expressing cell lysates of PC12 cells. Immunoblotting was performed with indicated antibodies. (D) PC12 cells were co-transfected with flag-tagged beta tubulin and a series of GFP-Ebp1 constructs as indicated. After 48 hours, cell lysates were subjected to immunoprecipitation assay against anti-flag antibodies, and immunoblotting was performed with indicated antibodies. distal axon. However, the anti-N-p48 antibody signal was notably strong in the distal growing neurite growth cone, suggesting that p48 Ebp1 expression is spatially and temporally correlated between subcellular locations and the growth cone during development (Supplementary Fig. 2). In the stage III hippocampal neuron, we found prominent endogenous Ebp1 expression in the growth cone central domain where microtubules were highly enriched and was co-distributed with Tuj1-labeled beta tubulin in distal microtubules (Fig. 3A).

Free tubulin dimers bind two GTP molecules, one at the non-exchangeable alpha tubulin $\mathrm{N}$-site and the other at the exchangeable beta tubulin E-site. Beta tubulin along the microtubule length is bound to GDP, and the plus end is capped by a GTP-bound beta tubulin layer (21). Interestingly, using differentiated cell lysates (DIV 2) from PC12 cells with purified GST-p48 and p42 proteins, we found that both Ebp1 isoforms bound to endogenous beta tubulin but not alpha tubulin (Fig. 3B). Reciprocally, we confirmed the specificity of this interaction using purified beta tubulin and GFP-p48- or GFP-p42-expressing cell lysates, showing the association between beta tubulin and both Ebp1 isoforms (Fig. 3C). Our mapping analysis showed that the Ebp1 $\mathrm{N}$-terminal domain (1-183) was sufficient and necessary for the interaction between Ebp1 and beta tubulin (Fig. 3D). Taken together, this data indicates that Ebp1 acts as an MAP and is involved in microtubule dynamics during axon growth.

\section{P48 Ebp1 contributes to axon regeneration in injured hippocampal slices}

Because we found that p48 Ebp1 was a microtubule-binding protein localized to the distal growth cone in growing axons, but its expression decreased after birth, we wondered whether p48 Ebp1 reintroduction to damaged brain would stimulate
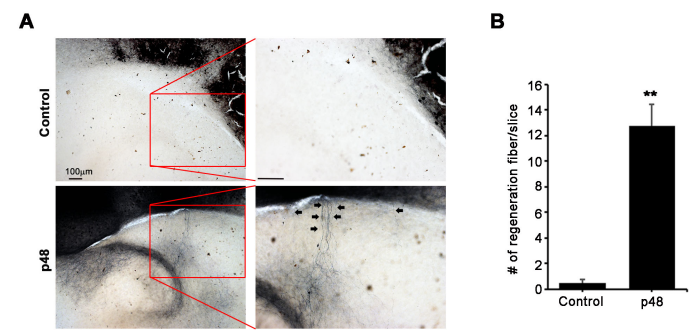

Fig. 4. P48 Ebp1 contributes to axon regeneration in injured hippocampal slices. Hippocampal slices were axotomized at the perforant path (PP) after DIV 7, and AAV2-p48Ebp1 or control virus was infected on DIV 9. Slices were cultured for an additional 14 days. The anterograde axonal tracer biocytin was placed on the entorhinal cortex. Biocytin was visualized using the ABC-DAB method. (A) Representative images of biocytin tracing (A) and quantification of regenerating axons (B). Black arrows indicate regenerated fibers. Scale bar, $100 \mu \mathrm{m}$. $* * P<0.005$ versus control. Images shown are representative of at least three independent experiments, and each value represents the mean \pm SEM of triplicate measurements. 
axon regeneration. After EH-OSC for 9 days, we introduced control AAV2 or AAV2-p48 into injured slices and maintained tissue culture for another 14 days. We used biocytin labeling for neuronal tissues and diaminobenzidine (DAB)-based processing for brain slices to determine if axons would regenerate. In agreement with a previous report (22), we saw negligible axon regrowth in control virus-infected slices post injury. However, both regenerating hippocampal axon number and length were notably increased in AAV2-p48 Ebp1-infected slices (Fig. 4A, B). This suggests that p48 Ebp1 introduction after CNS injury could promote axon regeneration and is perhaps a useful therapeutic approach for axotomy.

\section{DISCUSSION}

This study investigated Ebp1 isoform expression profile in the developing mouse brain. P48 Ebp1 is a predominant isoform in the brain, and its mRNA and proteins are significantly more highly expressed in the developing embryo than in adults, fitting with earlier results showing that p48 Ebp1 is highly expressed in rapidly proliferating cells such as glioblastomas, although still much more abundant than p42 expression, which is visible after birth. Interestingly, p48 Ebp1 expression in the hippocampus and entorhinal cortex is prominent in neurons rather than astrocytes, implying neuron-specific roles of p48 Ebp1 in the developing brain. Moreover, we found that p48 Ebp1 is enriched in some axonal growth cone tips and notably colocalized with microtubules, as shown by our in vitro binding assay showing the physical interaction between the p48 $\mathrm{N}$-terminal domain and beta tubulin. Furthermore, p48 Ebp1 overexpression in injured hippocampal slices elicited robust axon regeneration. Thus, these results suggest that p48 Ebp1 possesses microtubule-binding affinity and is possibly involved in its dynamics, thereby contributing to axon regeneration after injury in the $\mathrm{CNS}$.

Since Ebp1 was first discovered to be an interacting partner with the ErbB3 receptor, it has been considered a part of ErbB3 signaling. However, we have identified two Ebp1 isoforms, p48 and p42, and have shown that the long p48 Ebp1 isoform does not bind to ErbB3 and does not respond to neuregulin treatment (data not shown). Rather, it enhances Akt activity post-neurotrophin signaling including NGF and BDNF (18), whereas the short p42 Ebp1 isoform binds to ErbB3 during EGF treatment (6). Further support for ErbB3-independent p48 Ebp1 effects was shown during embryonic myoblast and adult stem cell proliferation and differentiation, indicating that Ebp1 down-regulation reduced myoblast proliferation and differentiation. These observations indicate that $\mathrm{p} 48$ acts as a general signaling molecule independent of ErbB3, and p42 may be a key binding partner for ErbB3 signaling. P42 invisible expression during the embryonic stage was correlated with the ErbB3 expression profile. ErbB3 mRNA is extremely limited in the developing mouse CNS. At E13, ErbB3 mRNA was not detectable and by $\mathrm{P} 7$, the signal was visible and maintained in adult major white matter (23). Nevertheless during brain development, ErbB1 and ErbB4 played more pivotal roles than ErbB2 and ErbB3 for mediating early postnatal development (24). Thus, although p42 mRNA and protein start to be detectable during the postnatal development period, the major Ebp1 isoform in the brain is probably p48 Ebp1 which is reduced developmentally in the hippocampus and cortex, reflecting its specific roles in early brain development.

Axon regeneration after injury relies on microtubule cytoskeletal reconstruction, reorganization, and stabilization at the axonal tip, where a balance between polymerization and depolymerization is critical for normal axon growth $(25,26)$. Growing numbers of microtubule regulators have been identified, but little information on how they regulate microtubule dynamics after injury has been generated. In developing neurons, p48 Ebp1 is localized to growing neurite tips, enriched in axonal growth cones, and shows almost complete colocalization with microtubules. Moreover, p48 Ebp1 associates with beta tubulin but not alpha tubulin, implying a possible p48 Ebp1 role in microtubule dynamics. Previously, we showed that p48 knockdown impaired neurite outgrowth in a rat hippocampal cell line (H19-7/IGF-IR) (18). This might be because p48 Ebp1 acts as an MAP to regulate microtubule dynamics in axon growth cones. Along with p48 Ebp1 effects on neurite elongation in the injured hippocampal slice, forced expression of p48 Ebp1 profoundly provoked axon regeneration. It will be worth identifying p48 Ebp1-specific mechanisms regulating microtubule dynamics and how they localize to dynamic microtubule tips in growth cones.

\section{MATERIALS AND METHODS}

\section{Primary neuron and cell culture}

E18 rat embryo brains were dissected, and hippocampi were removed and placed in a $15 \mathrm{ml}$ tube with $14 \mathrm{ml}$ of Hanks' Balanced Salt Solution (HBSS) on ice. Isolated hippocampi were cultured as previously described (18). HEK293T cells and PC12 cells were cultured as previously described (27).

\section{Reverse transcriptase polymerase chain reaction (RT-PCR)}

Brain lysates were obtained from E14-P21 mice. Total RNA was extracted with QIAzol ${ }^{\mathbb{R}}$ reagent (Qiagen, Valencia, CA, USA) according to the manufacturer's instructions. The reverse transcription reaction was performed using a Superscript II ${ }^{\mathrm{TM}}$ kit (Invitrogen). Expression of the two Ebp1 isoforms (Genbank accession number, NM 006191.2) was verified using GoTaq ${ }^{\mathbb{R}}$ Green Master mix (Promega, Madison, WI, USA). The RT-PCR primer sequences for Ebp1 were: (forward) 5'-ACAGCCTGTG GCTGGGAAGGG-3' (nucleotides 343 to 363), (reverse) 5'CTTCAAAGGGGAGAAGTG-3' (nucleotides 681 to 698) (product size, 356 and 290 bp).

\section{Immunofluorescence}

Immunostaining was performed as previously described (28, 
29) with the following modifications. Cells grown on coverslips in 24-well plates were fixed in 4\% paraformaldehyde for 15 min, permeabilized in PBS containing $0.25 \%$ Triton X-100 for $10 \mathrm{~min}$, and blocked in 1\% BSA for $30 \mathrm{~min}$. Cells were immunostained using primary antibodies and the appropriate Alexa Fluor 594 goat anti-rabbit and Alexa Fluor 488 goat anti-mouse secondary antibodies. Nuclei were counterstained with DAPI stain. Immunostained images were acquired using a laser scanning confocal microscope (LSM 710, Carl Zeiss, Germany). The confocal microscope was controlled using ZEN software, and the acquisition was performed in the Research Core Facility, SBRI.

\section{Mouse hippocampal slice culture}

Hippocampal slice cultures were prepared from P7 mouse brains. The $300-\mu \mathrm{m}$-thick brain slices were obtained by vibratome sectioning (Leica VT1200, Leica Biosystems) in chilled MEMp [50\% (vol/vol) minimum essential medium (MEM), $25 \mathrm{mM}$ HEPES, and $2 \mathrm{mM}$ glutamine without antibiotics, adjusted to $\mathrm{pH}$ 7.2-7.3 with $1 \mathrm{M} \mathrm{NaOH}$ ]. The slices were transferred onto semi-porous membrane inserts (Millipore, $0.4 \mu \mathrm{m}$ pore diameter, Schwalbach, Germany). Intact slices were cultured at $37^{\circ} \mathrm{C}$ and $5 \% \mathrm{CO}_{2}$ in a standard medium MEMi $[50 \%$ ( $\mathrm{vol} / \mathrm{vol})$ MEM, $25 \mathrm{mM}$ HEPES, 25\% ( $\mathrm{vol} / \mathrm{vol})$ HBSS, 25\% (vol/vol) heat-inactivated horse serum, $2 \mathrm{mM}$ glutamine, $1 \mathrm{ml}$ penicillin/streptomycin solution, and $0.044 \%$ ( $\mathrm{vol} / \mathrm{vol}$ ) $\mathrm{NaHCO}_{3}$, adjusted to $\mathrm{pH}$ 7.2-7.3 with $1 \mathrm{M} \mathrm{NaOH}$.] The medium was changed every other day. Hippocampal slices were either applied for immunostaining (Fig. 2) or axotomized (Fig. 4) after DIV 7, and AAV2s were used for infection at DIV 9. Slices were cultured for an additional 14 days. Biocytin anterograde axonal tracer was placed on the entorhinal cortex at DIV 21. Hippocampal slices were fixed with $4 \%$ PFA at DIV 22. Biocytin was visualized using the ABC-DAB method.

\section{Statistical analysis}

Data are expressed as mean \pm SEM of triplicate measurements from three independent experiments. Statistical analysis was performed using Sigmaplot Statistical Analysis Software (Systat software). All studies were performed in a blinded manner. Statistical significance was defined by Student's t-test $\left({ }^{*} \mathrm{P}<\right.$ $0.05 ; * * P<0.005)$.

\section{ACKNOWLEDGEMENTS}

This work was supported by a National Research Foundation of Korea (NRF) grant funded by the Korean government (MSIP) (NRF-2013R1A2A2A01005324) to J.-Y. Ahn and by a Samsung Biomedical Research Institute grant; grant number: SMX1161311, by Basic Science Research Program through the National Research Foundation of Korea(NRF) funded by the Ministry of Education(2016R1A6A3A11931078) to H.R. Ko.

\section{CONFLICTS OF INTEREST}

The authors have no conflicting financial interests.

\section{REFERENCES}

1. Radomski N and Jost E (1995) Molecular cloning of a murine cDNA encoding a novel protein, p38-2G4, which varies with the cell cycle. Exp Cell Res 220, 434-445

2. Yoo JY, Wang XW, Rishi AK et al (2000) Interaction of the PA2G4 (EBP1) protein with ErbB-3 and regulation of this binding by heregulin. British Journal of Cancer 82, 683-690

3. Figeac N, Serralbo O, Marcelle C and Zammit PS (2014) ErbB3 binding protein-1 (Ebp1) controls proliferation and myogenic differentiation of muscle stem cells. Dev Biol 386, 135-151

4. Yamada $\mathrm{H}$, Mori $\mathrm{H}$, Momoi $\mathrm{H}$, Nakagawa $\mathrm{Y}$, Ueguchi $\mathrm{C}$ and Mizuno T (1994) A fission yeast gene encoding a protein that preferentially associates with curved DNA. Yeast 10, 883-894

5. Ahn JY, Liu X, Liu Z et al (2006) Nuclear akt associates with PKCphosphorylated Ebp1, preventing DNA fragmentation by inhibition of caspase-activated DNase. EMBO J 25, 2083-2095

6. Liu Z, Ahn JY, Liu X and Ye K (2006) Ebp1 isoforms distinctively regulate cell survival and differentiation. Proc Natl Acad Sci U S A 103, 10917-10922

7. Liu Z, Oh SM, Okada M et al (2009) Human BRE1 Is an E3 Ubiquitin Ligase for Ebp1 Tumor Suppressor. Mol Biol Cell 20, 757-768

8. Monie TP, Perrin1 AJ, Birtley JR et al (2007) Structural insights into the transcriptional and translational roles of Ebp1. EMBO J 26, 3936-3944

9. Bose SK1, Sengupta TK, Bandyopadhyay S and Spicer EK (2006) Identification of Ebp1 as a component of cytoplasmic bcl-2 mRNP (messenger ribonucleoprotein particle) complexes. Biochem J 15, 99-107

10. Kim CK, Nguyen TLX, Joo KM et al (2010) Negative regulation of p53 by the long isoform of ErbB3 binding protein Ebp1 in brain tumors. Cacer Res 70, 9730-9741

11. Ko HR, Kim CK, Ahn JY et al (2015) Phosphorylation of the $\mathrm{N}$-terminal domain of $\mathrm{p} 48 \mathrm{Ebp} 1$ by CDK2 is required for tumorigenic function of p48. Mol Carcinog 54, 1283-1291

12. Mei Y, Zhang, Zuo H et al (2014) Ebp1 activates podoplanin expression and contributes to oral tumorigenesis. Oncogene 33, 3839-3850

13. Kim CK, Lee SB, Nguyen TL et al (2012) Long isoform of ErbB3 binding protein, p48, mediates protein kinase B/Akt-dependent HDM2 stabilization and nuclear localization. Exp Cell Res 318, 136-143

14. Xia X, Cheng A, Lessor T, Zhang $Y$ and Hamburger AW (2001) Ebp1, an ErbB-3 binding protein, interacts with Rb and affects $\mathrm{Rb}$ transcriptional regulation. J Cell Physiol 187, 209-217

15. Zhang Y, Wang XW, Jelovac D et al (2005) The ErbB3binding protein Ebp1 suppresses androgen receptormediated gene transcription and tumorigenesis of prostate 
cancer cells. PNAS 28, 9890-9895

16. Ko HR, Kim CK, Lee SB et al (2014) P42 Ebp1 regulates the proteasomal degradation of the $\mathrm{p} 85$ regulatory subunit of $\mathrm{PI} 3 \mathrm{~K}$ by recruiting a chaperone-E3 ligase complex HSP70/CHIP. Cell Death Dis 5, e1131

17. Hwang I, Kim CK, Ko HR et al (2016) C-terminal domain of p42 Ebp1 is essential for down regulation of p85 subunit of PI3K, inhibiting tumor growth. Sci Rep 6, 30626

18. Kwon IS and Ahn JY (2011) p48 Ebp1 acts as a downstream mediator of Trk signaling in neurons, contributing neuronal differentiation. Neurochem Int 58, 215-223

19. Perk J, lavarone A and Benezra R (2005) Id family of helix-loop-helix proteins in cancer. Nat Rev Cancer 5, 603-614

20. Sun $X$, Yao H, Douglas RM, Gu XQ, Wang J and Haddad GG (2010) Insulin/PI3K signaling protects dentate neurons from oxygen-glucose deprivation in organotypic slice cultures. J Neurochem 112, 377-388

21. Carlier MF, Hill TL and Chen YD (1984) Interference of GTP hydrolysis in the mechanism of microtubule assembly: An experimental study. Proc Natl Acad Sci U S A 81, 771-775

22. R' Io MR and DeFelipe J (1997) Synaptic Connections of Calretinin-Immunoreactive Neurons in the Human Neocortex. J Neurosci 17, 5143-5154
23. Fox IJ and Kornblum HI (2005) Developmental profile of ErbB receptors in murine central nervous system: implications for functional interactions. J Neurosci Res 79, 584-597

24. Chong VZ, Webster MJ, Rothmond DA, Weickert CS et al (2008) Specific developmental reductions in subventricular zone ErbB1 and ErbB4 mRNA in the human brain. Int J Dev Neurosci 26, 791-803

25. Conde C and Caceres A (2009) Microtubule assembly, organization and dynamics in axons and dendrites. Nat Rev Neurosci 10, 319-332

26. Bradke F, Fawcett JW, Spira ME et al (2012) Assembly of a new growth cone after axotomy: the precursor to axon regeneration. Nat Rev Neurosci 13, 183-193

27. Ahn JY, Rong R, Kroll TG et al (2004) PIKE (Phosphatidylinositol 3-Kinase Enhancer)-A GTPase Stimulates Akt Activity and Mediates Cellular Invasion. J Biol Chem 279, 16441-16451

28. Choi SH, Park BK and Lee KW (2015) Effect of respiratory syncytial virus on the growth of hepatocellular carcinoma cell-lines. BMB Rep 48, 565-570

29. Kim YJ, Kim JE, Choi HC, Song HK and Kang TC (2015) Cellular and regional specific changes in multidrug efflux transporter expression during recovery of vasogenic edema in the rat hippocampus and piriform cortex. BMB Rep 48, 348-353 